\section{Associação entre a qualidade de vida das mães e o estado nutricional de seus filhos}

\section{Association between mothers' quality of life and infants' nutritional status}

\section{Fernanda de Matos Feijó' \\ Deborah Filippini Carraro" \\ Maria Rita Macedo Cuervo"' \\ Martine Elisabeth Kienzle Hagen'v \\ Wilson Paloschi Spiandorellov}

\section{Alessandra Campani Pizzato ${ }^{\mathrm{VI}}$}

' Programa de Pós-Graduação em Medicina: Ciências Médicas da Universidade Federal do Rio Grande do Sul (UFRGS); Sistema de Saúde Mãe de Deus.

" Programa de Pós-Graduação em Ciências Cirúrgicas da Universidade Federal do Rio Grande do Sul (UFRGS).

III Programa de Pós Graduação em Psicologia da Pontifícia Universidade Católica do Rio Grande do Sul (PUCRS); Curso de Graduação em Nutrição da Faculdade de Enfermagem, Nutrição e Fisioterapia da Pontifícia Universidade Católica do Rio Grande do Sul - FAENFI/PUCRS.

Iv Curso de Graduação em Nutrição da Universidade Federal do Rio Grande do Sul (UFRGS).

v Curso de Graduação em Medicina da Universidade de Caxias do Sul (UCS).

vI Curso de Graduação em Nutrição da Faculdade de Enfermagem, Nutrição e Fisioterapia da Pontifícia Universidade Católica do Rio Grande do Sul - FAENFI/ PUCRS.

\section{Resumo}

Objetivo: Verificar associações entre a qualidade de vida das mães e o estado nutricional de seus filhos. Métodos: Foi realizado um estudo caso-controle com mães de crianças com idade entre zero e cinco anos, moradoras da área de abrangência de uma unidade básica de saúde, no município de Porto Alegre. O cálculo de tamanho amostral foi estimado em 152 mães, sendo 76 mães com filhos em risco nutricional/ desnutrição (casos) e 76 mães com filhos eutróficos (controles). Foram coletadas informações referentes à qualidade de vida das mães, medida através do instrumento da Organização Mundial da Saúde, e analisada a associação entre a qualidade de vida materna e o estado nutricional de seus filhos. Resultados: Em relação ao domínio psíquico, para cada criança eutrófica cuja mãe tem baixa qualidade de vida existe uma chance de 5,4 crianças em risco nutricional/ desnutrição com mães em igual condição. No domínio ambiental, para cada criança eutrófica cuja mãe tem baixa qualidade de vida existe uma chance de 2,9 crianças em risco nutricional/desnutrição com mães em igual condição. Em relação ao nível educacional, para cada criança eutrófica cuja mãe tem baixa qualidade de vida existe uma chance de 4,2 crianças em risco nutricional/desnutrição com mães em igual condição. Conclusões: A baixa qualidade de vida materna mostrou-se associada ao risco nutricional/desnutrição infantil e pode ser um fator de risco para o estado nutricional dos filhos.

Palavras-chave: Transtornos da nutrição infantil. Vigilância nutricional. Fatores de risco. Desnutrição. Qualidade de vida. Estado nutricional. 


\section{Abstract}

Objective: Determine associations between the quality of life of mothers and the nutritional status of children. Methods: case-control study involving 152 mothers of children aged zero to five years, living in the coverage area of a basic health unit in the city of Porto Alegre. The calculation of sample size was estimated as 152 mothers 76 mothers with children at nutritional risk/ malnutrition (cases) and 76 mothers with eutrophic children (controls). Information was collected regarding the quality of life of mothers, measured by the instrument of the World Health Organization, and the association between maternal quality of life and nutritional status of children was examined. Results: In relation to the psychiatric realm, for each eutrophic child whose mother has lower quality of life there is a chance of 5.4 children at nutritional risk/malnutrition with mothers in the same condition. In the environmental field, for each eutrophic child whose mother has lower quality of life there is a chance of 2.9 children at nutritional risk/malnutrition with mothers in the same condition. Regarding educational level, for each eutrophic child whose mother has lower quality of life there is a chance of 4.2 children at nutritional risk/malnutrition with mothers in the same condition. Conclusions: Mothers' low quality of life was associated with an infant in nutritional risk/ malnutrition and may be a risk factor for the nutritional status of children.

Keywords: Infant nutrition disorders. Nutritional vigilance. Risk factors. Malnutrition. Quality of life. Nutritional status.

\section{Introdução}

A desnutrição infantil é uma doença de origem multicausal e complexa com condicionantes biológicos e sociais, relacionada ao atendimento de necessidades básicas como saúde, alimentação, saneamento e educação, entre outros, e tem suas raízes na pobreza ${ }^{1-6}$. Percebe-se que a origem da desnutrição é multicausal quando esta se apresenta de forma diferenciada nas crianças de uma mesma família, entre famílias diversas e em classes sociais distintas ${ }^{6,7}$. Ou seja, mesmo vivendo sob as mesmas condições, as pessoas apresentam estados nutricionais diferentes ${ }^{4,7-10}$. Segundo Mon$\mathrm{te}^{11}$, a natureza do vínculo mãe-filho, bem como as práticas de cuidado com a criança e suas condições de vida são importantes fatores na gênese da desnutrição.

Para compreender a origem da desnutrição é preciso um conhecimento mais aprofundado dos fatores de risco de desnutrição. São eles: baixo nível socioeconômico, abandono do aleitamento materno, saneamento básico ausente ou inadequado, desajustamento familiar, fraco vínculo mãe-filho, baixa escolaridade, baixa estimulação e baixo peso ao nascer ${ }^{7-10}$. Dentre os fatores de risco de desnutrição, o vínculo mãe-filho tem se demonstrado de grande importância. Muitos autores enfatizam a importância da condição mental e psicológica da mãe como fator de risco para desnutrição $0^{4,8,12,13}$.

A boa relação entre mãe e filho é aquela em que há possibilidade de trocas afetivas. Para que esta relação se desenvolva, é necessário que a mãe possa exercer bem o seu papel materno, que é influenciado por vários fatores, tais como sua história de vida, os cuidados e afetos recebidos de seus pais e a qualidade da relação conjugal e familiar. Quando a mãe está bem assistida, tanto em relação à sua saúde quanto emocionalmente, ela desenvolve a função materna adequadamente $^{8,13,14}$. Isso nos leva a reconhecer que a qualidade de vida (QV) da mãe tem uma forte relação com o estado nutricional de seu filho, e que a mesma tem um impacto importante sobre a saúde dele $\mathrm{e}^{14,15}$. 
Reduzir a prevalência da desnutrição infantil requer ação focalizada e sistemática não apenas na área de saúde, mas também na segurança alimentar e, particularmente, nos cuidados com a mãe para que ela possa cuidar bem da sua criança ${ }^{6,11}$. Por isso, delineou-se o presente estudo a fim de verificar associações entre a $Q V$ das mães e o estado nutricional de seus filhos. A hipótese a ser testada foi de que mães com baixa QV apresentam maior chance de terem filhos em risco nutricional/desnutrição em relação a mães com QV aceitável. Utilizou-se o instrumento próprio para medir a QV da OMS e classificou-se as mães como em baixa QV e QV aceitável.

\section{Métodos}

Foi realizado no ano de 2006 um estudo de caso-controle com mães de crianças com idade entre zero e cinco anos, moradoras na área de abrangência de uma unidade básica de saúde, no município de Porto Alegre, em uma região que apresenta elevada vulnerabilidade social.

O cálculo de tamanho amostral foi estimado em 152 mães, sendo 76 mães com filhos em risco nutricional/desnutrição (casos) e 76 mães com filhos eutróficos (controles). Este número foi calculado para se obter um intervalo de confiança de $95 \%$, poder de $80 \%$ e odds ratio (OR) de três, valores esses considerados de relevância clínica pelos pesquisadores.

As participantes desse estudo foram selecionadas através do banco de dados de um estudo de vigilância nutricional, no ano de 2006, que realizou rastreamento do estado nutricional de todas as crianças com idade entre zero e cinco anos, moradoras na área de abrangência de uma unidade básica de saúde, no município de Porto Alegre ${ }^{17}$. Esta pesquisa, financiada pelo Conselho Nacional de Pesquisa e Desenvolvimento Científico e Tecnológico (CNPq), foi uma parceria entre a Pontifícia Universidade Católica do Rio Grande do Sul (PUCRS) e a Associação Hospitalar Moinhos de Vento (HMV). Este estudo realizou avaliação nutri- cional das crianças, empregando os índices antropométricos: peso/altura, altura/idade e peso/idade, sendo comparadas às curvas de referência do Center for Disease Control and Prevention (CDC) Growth Charts 2000, com dados do National Center for Health Statistics (NCHS), mediante a colaboração do National Center for Disease and Health Promotion $^{18,19}$. O peso corporal foi verificado por meio de uma balança da marca Plena, modelo MEA-07400, com capacidade para $150 \mathrm{~kg}$, devidamente calibrada, com a criança descalça e com o mínimo de roupa possível. A mensuração da estatura foi feita com a utilização do antropômetro horizontal (marca Sunny) para avaliação das crianças menores de 2 anos e com a utilização do antropômetro vertical para crianças acima de 2 anos (marca Sunny), descalças e com o mínimo de roupa possível.

Foram classificadas como risco nutricional/desnutrição aquelas crianças que apresentaram percentil menor que $10 \mathrm{em}$ dois desses índices, sendo um deles peso/ idade, e como eutróficas aquelas que apresentaram percentil entre 97 e 100, inclusive nos índices peso/altura e altura/idade ${ }^{1,15,16}$.

Assim, foram identificadas mães com filhos em risco nutricional/desnutrição e mães com filhos eutróficos. Todas as mães que apresentaram pelo menos um filho em risco nutricional ou desnutrido foram incluídas no caso. Foram excluídas do estudo as mães que estavam grávidas no momento da avaliação, as que possuíam filhos em tratamento de qualquer doença crônica, como também as que não quiseram participar do estudo.

Foi realizada alocação aleatória das participantes através de um software de informática do banco de dados da pesquisa de vigilância nutricional acima referido. Foram coletadas informações referentes à QV das mães, medidas através do instrumento de avaliação da QV, oWHOQOL-bref, criado pela Organização Mundial da Saúde (OMS) e validado no Brasil ${ }^{20}$. Trata-se de um questionário com vinte e seis questões que foi desenvolvido com a perspectiva de ser transcultural, ou seja, considerando as 
diferenças culturais para poder ser utilizado internacionalmente. Empregando uma escala com valores que variam entre zero e 100, avaliam-se quatro diferentes domínios: físico (sete questões), psicológico (seis questões), relações sociais (três questões), meio ambiente (oito questões), além de duas questões adicionais sobre QV geral. Este instrumento estima as condições em que a pessoa se encontrava nos últimos 15 dias $^{21}$. Esta versão resumida é uma alternativa para as situações em que a versão longa seja considerada de difícil aplicação, como, por exemplo, em estudos com utilização de múltiplos instrumentos de avaliação ${ }^{20,21}$.

Ele se origina do WHOQOL-100, constituído por 100 questões que verificam as condições em que a pessoa se vê, percebe, considera e se analisa, ao longo dos 15 dias precedentes. As questões foram formuladas para respostas em uma escala de: intensidade (nada - extremamente), capacidade (nada-completamente), frequência (nunca - sempre) e avaliação (muito insatisfeito muito satisfeito; muito bom - muito ruim), cujos escores são em uma direção positiva, isto é, escores maiores denotam melhor $\mathrm{QV}^{22-24}$.

Segundo Fleck e colaboradores, o WHOQOL-bref apresenta boa consistência interna, validade discriminante, validade concorrente, validade de conteúdo e confiabilidade teste-reteste. A consistência interna doWHOQOL-bref, medida pelo coeficiente a de Cronbach, foi boa; quando comparada com a consistência interna da versão longa de 100 questões, o coeficiente de Cronbach foi inferior. Segundo os autores, este achado era esperado, pois instrumentos compostos por um número maior de questões tendem a ter coeficientes de Cronbach mais eleva$\operatorname{dos}^{20}$.

Avaliar a QV é muito complexo, uma vez que não existe uma definição consensual sobre o que ela realmente significa. $\mathrm{O}$ conceito que norteia o trabalho é o mesmo adotado pela OMS, definido como "a percepção do indivíduo sobre sua posição na vida, no contexto da cultura e dos sistemas de valores nos quais ele vive, e em relação a seus objetivos, expectativas, padrões e preocupações" ${ }^{25}$.

O WHOQOL-bref foi escolhido por ser um instrumento genérico que procura englobar todos os aspectos importantes relacionados à saúde, e que reflete o impacto de uma doença sobre o indivíduo. Pode ser usado para estudar indivíduos da população geral ou de grupos específicos, como portadores de doenças crônicas. Assim, permite comparar a QV de indivíduos sadios com doentes ou portadores da mesma doença, vivendo em diferentes contextos sociais e culturais $^{25,26}$.

Inicialmente, os resultados foram descritos para toda a amostra e, em seguida, para os grupos em separado. Depois, os domínios de QV foram comparados, nos grupos, segundo o estado nutricional: risco nutricional/desnutrição e eutrofia, através do teste $t$ de Student. Para a análise multivariada de regressão logística, o nível educacional foi categorizado em primeiro grau e outros, sendo esse último a agregação do segundo e terceiro graus. Os motivos para se realizar esse tipo de transformação foram a relação entre o tamanho da amostra e o número excessivo de categorias, e a perda irrelevante de informações para os objetivos do estudo.

Os domínios foram classificados em qualidade aceitável, com escores acima de $60 \%$, e baixa qualidade, com escore inferior. Esses valores foram identificados como um ponto de corte diferencial durante as análises estatísticas.

Os princípios éticos foram respeitados com o objetivo de proteger os direitos dos sujeitos envolvidos, considerando-se as questões expressas na Resolução 196/96 do CNS-MS ${ }^{27}$, as recomendadas por Polit e Hungler ${ }^{28}$, e os aspectos apontados por Goldim $^{29}$. O projeto de pesquisa foi aprovado por dois Comitês de Ética: o Comitê de Ética em Pesquisa da PUCRS e do HMV.

\section{Resultados}

Na Tabela 1, observam-se as comparações entre os grupos de mães com filhos 
Tabela 1 - Valores estatisticamente significativos da comparação dos escores de QV entre os grupos caso e controle com o teste t de Student (Porto Alegre, 2006); p.11

Table 1 - Statistically significant comparison of QOL scores between case and control groups with Student's t test (Porto Alegre, 2006), p.11

\begin{tabular}{lccc}
\hline Domínio & Grupos & $\mathrm{M}^{2} \mathrm{DP}^{3}$ & $\mathrm{p}^{4}$ \\
\hline Físico & Caso $^{1}$ & $74,86 \pm 13,309$ & 0.04 \\
& Controle $^{2}$ & $80,88 \pm 12,292$ & \\
Psíquico & Caso $^{1}$ & $61,28 \pm 16,614$ & 0.00 \\
& Controle $^{2}$ & $73,38 \pm 10,302$ & \\
Social & Caso $^{1}$ & $66,09 \pm 17,475$ & 0.01 \\
& Controle $^{2}$ & $74,70 \pm 12,210$ & \\
Ambiental & Caso $^{1}$ & $52,53 \pm 13,218$ & 0.00 \\
& Controle $^{2}$ & $65,04 \pm 10,401$ & \\
\hline
\end{tabular}

'Caso: mães de crianças em risco nutricional/desnutrição; ${ }^{2}$ Controle: mães de crianças eutróficas; ${ }^{3} \mathrm{M} \pm \mathrm{DP}$ : Média \pm desvio padrão; ${ }^{4}$ Valores com significância estatística no teste t de Student ( $p>0.05$ ).

${ }^{1}$ Case: mothers of children at malnutrition/nutritional risk; ${ }^{2}$ Control: mothers of normal children; ${ }^{3} \mathrm{M} \pm D P$ : Average \pm deviation; ${ }^{4} \mathrm{Val}-$ ues with statistical significance in Student's t test $(p>0.05)$.

eutróficos e mães com filhos em risco nutricional/desnutrição quanto aos escores de QV. Analisando os domínios de QV (físico, psíquico, social e ambiental) dos grupos caso e controle, com teste t de Student, todos foram estatisticamente significativos.

O nível educacional foi analisado por uma variável dicotômica que representou aqueles com nível primário e os outros (agregação do segundo e terceiro graus) (Tabela 2). Essa classificação foi feita pelo número insuficiente de pessoas com educação de nível superior (quatro sujeitos). Das 152 mães, 91 (59,9\%) possuíam I grau incompleto; 17 (11,2\%) I grau completo; 19 (12,5\%) II grau incompleto; 21 (13,8\%) II grau completo, e 4 (2,6\%) III grau incompleto. Das mães que tinham I grau incompleto $34(37,4 \%)$ eram do grupo controle e 57 $(62,6 \%)$ do grupo caso. Com o I grau completo, $8(41,1 \%)$ controle e $9(52,9 \%)$ caso. Com II grau incompleto, 13 (68,4\%) controle e $6(31,6 \%)$ caso. Com o II grau completo, 18 (85,7\%) controle e $3(14,3 \%)$ caso. E com III grau incompleto, 3 (75\%) controle, e 1 (25\%) caso. $(\mathrm{p}=0.01)$.

A Tabela 3 apresenta a análise multivariada de regressão logística dos domínios

Tabela 2 - Distribuição da variável nível educacional entre os grupos caso e controle (Porto Alegre, 2006); p.11

Table 2 -Distribution of the educational level variable between case and control groups (Porto Alegre, 2006), p.11

\begin{tabular}{lccc}
\hline Nível educacional & Caso $^{1}$ & Controle $^{2}$ & Total \\
\hline I grau incompleto & $57(62,6 \%)$ & $34(37,4 \%)$ & 91 \\
I grau completo & $9(52,9 \%$ & $8(47,1 \%)$ & 17 \\
II grau incompleto & $6(31,6 \%)$ & $13(68,4 \%)$ & 19 \\
II grau completo & $3(14,3 \%)$ & $18(85,7 \%)$ & 21 \\
III grau incompleto & $1(25 \%)$ & $3(75 \%)$ & 4 \\
\hline Total & $76(50 \%)$ & $76(50 \%)$ & 152 \\
\hline
\end{tabular}

${ }^{1}$ Caso: mães de crianças em risco nutricional/desnutrição; ${ }^{2}$ Controle: mães de crianças eutróficas; Quanto ao nível educacional o grupo caso teve número maior de primeiro grau incompleto e menor de segundo grau $(p<0.01)$.

${ }^{1}$ Case: mothers of children at malnutrition/nutritional risk; ${ }^{2}$ Control: mothers of normal children; the educational level of the case group had higher number of individuals with incomplete elementary and lower secondary schooling $(p<0.01)$. 
Tabela 3 - Valores estatisticamente significativos da comparação dos escores de QV entre os grupos caso e controle através de análise multivariada de regressão logística (Porto Alegre, 2006); p.11

Table 3 - Statistically significant comparison of QOL scores between case and control groups through multivariate logistic regression (Porto Alegre, 2006), p.11

\begin{tabular}{lccc}
\hline Domínio & Grupos & $\mathrm{M} \pm \mathrm{DP}^{3}$ & $\mathrm{p}^{4}$ \\
\hline Físico & Caso $^{1}$ & $74,86 \pm 13,309$ & 0.04 \\
& Controle $^{2}$ & $80,88 \pm 12,292$ & \\
Psíquico & Caso $^{1}$ & $61,28 \pm 16,614$ & 0.00 \\
& Controle $^{2}$ & $73,38 \pm 10,302$ & \\
Social & Caso $^{1}$ & $66,09 \pm 17,475$ & 0.01 \\
& Controle $^{2}$ & $74,70 \pm 12,210$ & \\
Ambiental & Caso $^{1}$ & $52,53 \pm 13,218$ & 0.00 \\
& Controle $^{2}$ & $65,04 \pm 10,401$ & \\
\hline
\end{tabular}

${ }^{1}$ Caso: mães de crianças em risco nutricional/desnutrição; ${ }^{2}$ Controle: mães de crianças eutróficas; ${ }^{3} \mathrm{M} \pm \mathrm{DP}:$ Média \pm desvio padrão; ${ }^{4}$ Valores com significância estatística através de análise multivariada de regressão logística $(p>0.05)$.

${ }^{1}$ Case: mothers of children at malnutrition/nutritional risk; ${ }^{2}$ Control: mothers of normal children; ${ }^{3} \mathrm{M} \pm D P$ : Average \pm deviation; ${ }^{4} \mathrm{Val}-$ ues with statistical significance through multivariate logistic regression $(p>0.05)$.

de QV. Os escores de QV foram classificados como qualidade aceitável, quando acima de $60 \%$, e baixa qualidade, com escore inferior. Foram considerados: o estado civil, o nível educacional, o estado de saúde atual e os quatro domínios de QV. Por essa análise, a QV física e a social deixaram de mostrar significância estatística pela interferência das outras variáveis. Permaneceram estatisticamente significativos os odds ratio da qualidade psíquica, da qualidade ambiental e do nível educacional.

Em relação ao domínio psíquico, para cada criança eutrófica cuja mãe tem baixa QV existe uma chance de 5,4 crianças em risco nutricional/desnutrição com mães em igual condição. No domínio ambiental, para cada criança eutrófica cuja mãe tem baixa QV existe uma chance de 2,9 crianças em risco nutricional/desnutrição com mães em igual condição. Em relação ao nível educacional, para cada criança eutrófica cuja mãe tem baixa QV existe uma chance de 4,2 crianças em risco nutricional/desnutrição com mães em igual condição.

Na Tabela 4 observa-se a distribuição do estado civil entre os grupos. Das 152 mães, 35 (23\%) eram solteiras, 48 (31,6\%) eram casadas, $62(40,8 \%)$ viviam como casadas, 3 (2\%) eram separadas, $3(2 \%)$ eram divorciadas, e $1(0,7 \%)$ era viúva. Das mães solteiras, $15(42,9 \%)$ eram do grupo controle e $20(57,1 \%)$ do grupo caso. Casadas, 32 $(66,7 \%)$ eram do grupo controle e 16 (33,3\%) do grupo caso. Vivendo como casadas, 27 $(43,5 \%)$ eram controle e $35(56,5 \%)$ caso. Separadas, 1 (33,3\%) controle e $2(66,7 \%)$ caso. Divorciadas, $1(33,3 \%)$ controle e 2 (66,7\%) caso. E viúvas, 1 (100\%) era do grupo caso. $(\mathrm{p}=0.12)$.

Em relação à saúde das mães: estavam com a saúde muito ruim, 1 (50\%) de cada grupo; com a saúde fraca, $3(42,9 \%)$ do grupo controle e $4(57,1 \%)$ do grupo caso; com a saúde nem ruim e nem boa $16(38,1 \%)$ controle e 26 (61,9\%) caso; com a saúde boa 47 (56\%) controle e 37 (44\%) caso; com saúde muito boa $9(52,9 \%)$ controle e $8(47,1 \%)$ caso. De acordo com os problemas atuais de saúde dessas mães, tinham algum tipo de câncer: 1 (33,3\%) do grupo controle e 2 $(66,7 \%)$ do grupo caso; apresentaram Depressão: 6 (50\%) de cada grupo; Diabetes: $1(50 \%)$ era do grupo controle e $1(50 \%)$ do grupo caso; Doença de pele: $1(33,3 \%)$ controle e $2(66,7 \%)$ caso; Enfisema ou bronquite: $1(16,7 \%)$ no grupo controle e $5(83,3 \%)$ no grupo caso; Referindo não ter nenhum problema de saúde: $47(56,6 \%)$ controle e $36(43,4 \%)$ caso; Com outros problemas não 
Tabela 4 - Distribuição da variável estado civil entre os grupos caso e controle (Porto Alegre, 2006); p.12

Table 4 - Distribution of the marital status variable between case and control groups (Porto Alegre, 2006), p.12

\begin{tabular}{|c|c|c|c|}
\hline Estado civil & Caso $^{1}$ & Controle $^{2}$ & Total \\
\hline Solteira & $20(57,1 \%)$ & $15(42,9 \%)$ & 35 \\
\hline Casada & $16(33,3 \%)$ & $32(66,7 \%)$ & 48 \\
\hline Vivendo como casada & $35(56,5 \%)$ & $27(43,5 \%)$ & 62 \\
\hline Separada & $2(66,7 \%)$ & $1(33,3 \%)$ & 3 \\
\hline Divorciada & $2(66,7 \%)$ & $1(33,3 \%)$ & 3 \\
\hline Viúva & $1(100 \%)$ & $0(0 \%)$ & 1 \\
\hline Total & $76(50 \%)$ & $76(50 \%)$ & 152 \\
\hline
\end{tabular}

citados no questionário: 10 (40\%) controle e 15 (60\%) caso; Pressão alta: 5 (50\%) de cada grupo; Problemas de coração: 2 (66,7\%) controle e 1 (33,3\%) caso. Problema nervoso ou emocional: 1 (25\%) controle e $3(75 \%)$ caso. $(\mathrm{p}=0.617)$.

\section{Discussão}

A associação entre desnutrição infantil e o estado psíquico da mãe, nível educacional materno, bem como condições ambientais identificadas neste estudo, confirmaram resultados conhecidos na literatura. No estudo realizado por Cavalhaes \& Benício ${ }^{31}$ foram identificados como fatores de risco de desnutrição infantil a precária saúde mental materna, estrutura familiar adversa e fatores de estresse familiar. A baixa escolaridade mais que duplicou o risco de desnutrição. Reichenheim \& Harplan ${ }^{32}$ identificaram a condição econômica da família, indicada pelas condições ambientais de domicílio, um importante antecedente de déficit estatural. O grau de escolaridade materna também se mostrou associado ao retardo de crescimento ${ }^{3,8-10,33,34}$.

Marques $^{30}$, em um estudo realizado, avaliou a QV e sexualidade de mulheres em diálise observou resultado semelhante ao do presente estudo em relação à variável nível educacional, no qual a baixa escola- ridade teve efeito redutor da QV. Engstrom \& Anjos ${ }^{10}$ observaram maior incidência de desnutrição em filhos de mães analfabetas, reduzindo conforme o aumento da escolaridade. Resultado confirmado por Olinto et al. ${ }^{8}$ em seu estudo sobre os determinantes da desnutrição infantil em populações de baixa renda. Nascimento et al. ${ }^{9}$ deixam clara a relação entre depressão e vínculo mãe-filho comprometido, podendo levar à perda de interesse e à negligência ${ }^{3,31,34}$.

A baixa renda foi apontada em alguns estudos como um importante fator de risco para desnutrição ${ }^{3,33,34}$. Post et al. ${ }^{33}$ evidenciaram uma clara relação inversa entre a renda familiar e a prevalência de déficit. A renda familiar seria importante de ser considerada, entretanto, devido ao nível uniforme da população ser de baixa renda, ela não foi considerada neste estudo.

Alguns autores destacam o fato de mães que trabalham fora de casa, e que possuam renda mensal, como sendo significante para o estado nutricional infantil ${ }^{5,8}$. Segundo Olinto et al. ${ }^{8}$, que investigaram os determinantes de desnutrição infantil, as famílias cujas mães não trabalhavam fora de casa apresentaram um aumento de $70 \%$ no risco de déficit de altura/idade. Esta foi uma variável que poderia ter sido incluída. O número de irmãos também teve associação com o estado nutricional infantil. 
Reichenhrim \& Harpham ${ }^{32}$, em seu estudo com crianças menores de 5 anos em uma comunidade de baixa renda, identificaram que uma criança com pelo menos mais dois irmãos vivendo no mesmo domicílio tinha maior chance de crescimento retardado ${ }^{4}$.

Estudos também apontam a idade materna como um importante fator de risco para desnutrição infantil. Este foi um ponto fraco do presente estudo, pois a idade materna não foi avaliada ${ }^{3,31}$.

Para melhor esclarecer essa associação entre a baixa QV materna e risco nutricional/desnutrição infantil, novos estudos devem ser realizados utilizando-se mais de um instrumento de avaliação da QV ou a versão longa, oWHOQOL-100, bem como a inclusão das variáveis não analisadas neste estudo, como: idade materna, o fato de a mãe trabalhar (possuir renda própria) e a renda familiar.

\section{Conclusão}

Em resumo, os resultados da literatura estão de acordo com o presente estudo, sugerindo que a baixa QV materna está associada com o risco nutricional/desnutrição infantil e esta pode ser um fator de risco para o estado nutricional de seus filhos.

\section{Referências}

1. Vitolo MR. Nutrição: da gestação à adolescência. Rio de Janeiro: Reichmann \& Afonso Editores; 2003.

2. Cuervo MRM, Aerts DRG, Halpern R. Vigilância do estado nutricional das crianças de um distrito de saúde no sul do Brasil. J Pediatr 2005; 81: 325-31.

3. Machado MFAS, Vieira, NFC. Participação na perspectiva de mães de crianças desnutridas. Rev Latino-am Enfermagem 2004; 12(1): 76-82.

4. Nóbrega FJ. Atenção à Criança Desnutrida em áreas urbanas. São Paulo: Departamento de Nutrição/ Sociedade Brasileira de Pediatria 2001; p. 56-66.

5. Carvalho NM, Giugliani ERJ, Seffrin CF, Hartmann RM. Seguimentos de criança com desnutrição moderada ou grave em população periférica (Brasil). Rev Saúde Pública 1992; 26(4): 223-28.

6. Ieda MAP, Anézia MFM. Ter um filho desnutrido: O significado para as mães. Rev Min Enf 2006; 10(1): 12-17.

7. Rocha L, Gerhardt TE, Santos DL. Desnutrição e excesso de peso em crianças menores de 5 anos no meio rural de Arambaré, RS: (Des)construindo idéias repensando novos desafios. Cienc Cuid Saude 2007; 6(2): 206-14.

8. Olinto MTA, Victora CG, Barros FC, Tomasi E. Determinantes da desnutrição infantil em uma população de baixa renda: um modelo de análise hierarquizado. Cad Saúde Pública 1993; 9(1): 14-27.

9. Nascimento CL, Falcone VM, Spada PV, Mäder CV, Nóbrega FJ. Características psicológicas de mães de crianças desnutridas e a relação com o vínculo mãe/ filho. Rev Br Nutr Clín 2003; 18(3): 101-5.

10. Engstrom EM, Anjos LA. Déficit estatural nas crianças brasileiras: relação com condições sócio-ambientais e estado nutricional materno. Cad Saúde Pública 1999; 15(3): 559-67.
11. Monte C. Desnutrição: um desafio secular à nutrição infantil. J Pediatr 2000; 76(3): 285-97.

12. Fernandes BS. Nova abordagem para o grave problema da desnutrição infantil. Estud Av 2003; 17(48): 77-92.

13. Seidl EMF, Zannon CMLC. Qualidade de vida e saúde: aspectos conceituais e metodológicos. Cad Saúde Pública 2004; 20(2): 580-8.

14. Fleck MPA. O instrumento de avaliação de Qualidade de Vida da Organização Mundial da Saúde (WHOQOL-100): características e perspectivas. Ciênc Saúde Coletiva 2000; 5(1): 33-8.

15. Gouveia ELC. Nutrição, saúde e comunidade. 2 ed. Rio de Janeiro: Revinter; 1999.

16. Seidl EMF, Zannon CML da Costa. Qualidade de vida e saúde: aspectos conceituais e metodológicos. Cad Saúde Pública 2004; 20(2): 580-8.

17. Pizzato AC, Almeida CS, Cuervo MRM, Hagen MEK, Canabarro S. Vigilância e educação em saúde de crianças desnutridas e obesas da área adstrita a duas unidades básicas do município de Porto Alegre. Porto Alegre: Pontifícia Universidade Católica do Rio Grande do Sul; 2006 [não publicado].

18. National Center for Health Statistics. Developed by the National Center for Health Statistics in collaboration with the National Center for Chronic Disease Prevention and Health Promotion; 2000. Disponível em http:// www.cdc.gov/growthcharts [Acesso em 02/01/2010]

19. NCHS, 1977. NCHS Growth Curves for Children Birth-18 Years United States. Washington, DC: U.S. Printing Office. (Vital and Health Statistics Series 11, no. 165, DHEW Pub. no. 78-1650). 
20. Fleck MPA, Louzada S, Xavier M, et al Aplicação da versão em português do instrumento WHOQOL-Bref. Rev Saúde Pública 2000; 34(2): 178-83.

21. The Whoqol Group. Development of the World Health Organization WHOQOL-Brief Quality of Life assessment 1998. Psychol Med 1998; 28: 551-8.

22. Fleck MPA. O instrumento de avaliação de Qualidade de Vida da Organização Mundial da Saúde (WHOQOL-100): características e perspectivas. Ciênc Saúde Coletiva 2000; 5(1): 33-8.

23. Fleck MPA, Louzada S, Xavier M, et al. Desenvolvimento da versão em português do instrumento de avaliação de qualidade de vida da organização mundial da saúde (WHOQOL-100) 1999. Rev Brasileira de Psiquiatria 1999; 21(1): 19-28.

24. Fleck MPA, Louzada S, Xavier M, et al. Aplicação da versão em português do instrumento de avaliação de qualidade de vida da organização mundial da saúde (WHOQOL-100). Rev Saúde Pública 1999; 33(2): 198-205.

25. Seidl EMF, Zannon CML da Costa. Qualidade de vida e saúde: aspectos conceituais e metodológicos. Cad Saúde Pública 2004; 20(2): 580-8.

26. Dantas RAS, Sawada NO, Malerbo MB. Pesquisas sobre qualidade de vida: revisão da produção científica das universidades públicas do Estado de São Paulo. Rev Latino-Am Enfermagem. 2003; 11(4):532-538

27. Brasil CS. Diretrizes e normas regulamentadoras de pesquisa em seres humanos (Resolução 196/96). Diário Oficial da União. 16 de outubro de 1996.
28. Polit DF, Humgler BP. Fundamentos de pesquisa em enfermagem. Porto Alegre (RS): Artes Médicas; 1995.

29. Goldim JR. Manual de Iniciação à Pesquisa em Saúde. Porto Alegre (RS): DaCasa; 1997.

30. Marques FZC. Qualidade de vida e sexualidade de mulheres em diálise [dissertação de mestrado]. Porto Alegre: Faculdade de Medicina da Pontifícia Universidade Católica do Rio Grande do Sul.

31. Carvalhaes MBL, Benício MHD. Capacidade materna de cuidar e desnutrição infantil. Rev Saúde Pública 2002; 36(2): 188-97.

32. Reichenheim ME, Harpham T. Perfil intracomunitário da deficiência nutricional: estudo de crianças abaixo de 5 anos numa comunidade de baixa renda do Rio de Janeiro (Brasil). Rev Saúde Pública 1990; 24(1): 69-79.

33. Post CL, Victora CG, Barros FC, Horta BL, Guimarães PRV. Desnutrição e obesidade infantis em duas coortes de base populacional no sul do Brasil: tendências e diferenciais. Cad Saúde Pública 1996; 12(1): 49-57.

34. Raquel S, Evelise B, Ana Paula G, Simone B, Thaís LR, Carla AS. Avaliação do desenvolvimento neuropsicomotor em crianças de um bairro da periferia de Porto Alegre. Scientia Medica 2007; 17(3): 130-37.

Recebido em: 21/01/2010

Versão final apresentada em: 20/07/2010 Aprovado em: 11/04/2011 